\title{
Selective arterial embolization for a high-flow priapism following perineal trauma in a young gymnast
}

\author{
Grazia Bianchi $^{1}$, Camilla Sachs $^{2}$, Irene Campo ${ }^{2}$, Giovanni Liguori ${ }^{1}$, Carlo Trombetta ${ }^{1}$ \\ ${ }^{1}$ Department of Urology, University of Trieste, Cattinara Hospital, Trieste, Italy; \\ ${ }^{2}$ Department of Radiology, University of Trieste, Cattinara Hospital, Trieste, Italy.
}

\begin{abstract}
Summary Introduction. High-flow priapism is a rare condition in children, usually due to a perineal trauma.

Materials and methods. We present a case of traumatic highflow priapism investigated by Doppler ultrasound and managed by angiography and selective embolization of a branch of the internal pudendal artery.

Results. A 13-year-old gymnast underwent perineal trauma during training and developed a high-flow priapism. The first ultrasound (immediately after the trauma) showed the presence of an inhomogeneous area of $3 \times 2 \mathrm{~cm}$ associated with an anechoic vascularized area (pseudoaneurysm) in the right corpus cavernosum. On the left side there was a similar finding, but of smaller size. After 3 weeks the pseudoaneurysm on the left was completely obliterated while the right one was still present. Angiography and superselective catheterization of a branch of the left pudendal artery and its embolization with microspheres and with metal microcoils were performed. After the procedure, ultrasound showed that the right pseudoaneurysm was completely obliterated and there were no more branches reaching it. The cavernous arteries were both pervious.

Conclusions. Selective arterial embolization is a safe treatment that can also be used in pediatric patients.
\end{abstract}

KEY WORDS: High-flow priapism; Perineal trauma; Embolization.

Submitted 5 June 2018; Accepted 26 July 2018

\section{INTRODUCTION}

High-flow (non-ischaemic, arterial) priapism is a nonsexual, persistent erection caused by unregulated cavernous arterial inflow. Cavernous blood gases are not hypoxic or acidotic. Typically the penis is neither fully rigid nor painful. In children this is a rare condition, usually due to a perineal trauma and does not require emergent treatment (1).

\section{Case report}

We present the case of a 13-year-old prepubertal gymnast. He was performing an exercise on parallel bars. During a jump he hit a wooden bar with his genitalia, underwent perineal trauma and developed a high flow priapism. He had a scrotal hematoma, but he had no pain and his penis was not completely rigid. The first ultrasound (immediately after the trauma) showed, in the right corpus cavernosum, the presence of an inhomogeneous area of $3 \times 2 \mathrm{~cm}$, associated with an anechoic vascularized area (pseudoaneurysm) (2). On the left side there was a similar finding, but of lesser magnitude. After 3 weeks, the patient came to our attention and we performed another Doppler ultrasound using linear 5-12 and 5-17 $\mathrm{MHz}$ transducers. During the exam, a modest degree of erection could be appreciated. In correspondence to the palpatory finding in the crura, a $1.2 \times 0.4 \mathrm{~cm}$ cavernous pseudoaneurysm was recognized on the right, fed by a small fistula with turbulent flows and with high velocities. No other vascular afferents were recognized. With compression, we could stop the flow in the pseudoaneurysm with immediate resumption of the pathological stream at the end of compression. After 3 weeks, the pseudoaneurysm on the left was completely obliterated. After retrograde guidewire puncture of the left common femoral artery, we proceeded to selective catheterization of the right hypogastric artery and the right pudendal artery, which were found to be pervious. Angiography showed the presence of an arteriocavernosal fistula at the root of the penis with an afferent arterial branch originating from the pudendal artery. We proceeded to superselective catheterization of the branch of the right pudendal artery and its embolization with microspheres (Embozene $250 \mu \mathrm{m}$ ) and with metal microcoils (Striker $2 \times 40 \mathrm{~mm}$ ).

The final angiographic check demonstrated the complete occlusion of the embolized arterial branch (Figure 1).
Figure 1.

Angiography before and after embolization.
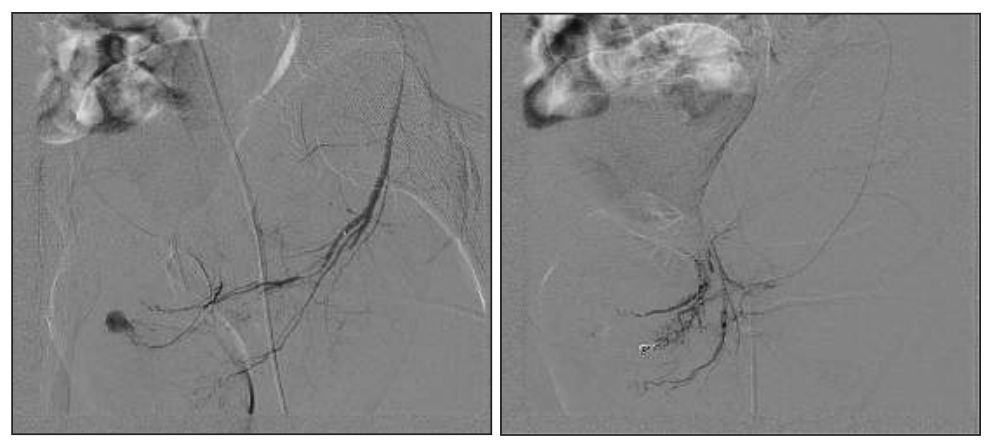

No conflict of interest declared. 
Afterwards, selective catheterization of the left hypogastric artery and of the left pudendal artery were performed and the angiography did't show any arteriocavernosal fistula on this side (3).

Ultrasound performed after the procedure showed that the right pseudoaneurysm was completely obliterated and there were no more branches reaching it. The cavernous arteries were both pervious.

\section{Conclusions}

Selective arterial embolization is a safe and painless treatment that can also be used in young patients.

\section{References}

1. Hacker HW, Schwoebel MG, Szavay PO. Nonischemic priapism in childhood: a case series and review of literature. Eur J Pediatr Surg. 2018; 28:255-260.

2. Vega-Vigo C, Márquez-Moreno AJ, Rojo-Carmona LE, Castillo Gallardo E. High-flow priapism caused by a pseudoaneurysm and an arteriocavernosal fistula: clinical and radiological approach of 3 cases. Arch Esp Urol. 2014; 67:642-5.

3. Bertolotto M, Quaia E, Mucelli FP, et al. Color Doppler imaging of posttraumatic priapism before and after selective embolization. Radiographics 2003; 23:495-503.

\section{Correspondence}

Grazia Bianchi, MD (Corresponding Author)

graziuccia88@libero.it

Giovanni Liguori, MD

gioliguori33@gmail.com

Carlo Trombetta, MD

trombcar@units.it

Università degli Studi Trieste, Urology Department - Cattinara Hospital,

Strada di Fiume 447, Trieste, Italy

Camilla Sachs, MD

cami_sachs@hotmail.it

Irene Campo, MD

irenecampol1@gmail.com

Università degli Studi Trieste, Radiology Department - Cattinara Hospital,

Trieste, Italy 